\title{
Photographic recording of slit-lamp appearances of the ocular fundus
}

\author{
PETER KENYERES AND HANS SLEZAK \\ From the Second Eye Clinic of the University of Vienna, and the Eye Department of the \\ Schwerpunktkrankenhaus Mistelbach, Lower Austria
}

SUMmARY The four methods of illumination in the use of slit-lamp biomicroscopy of the anterior segment, described first by Vogt, are equally useful in the posterior part of the eye. Examination of the ocular fundus is more difficult. In particular, photography of the central and peripheral parts of the fundus demands additional technical arrangements if all the appearances of biomicroscopy are to be recorded.

A major task of medical photography is to record as clearly as possible all the optical details revealed at various examinations.

Each method of examination has to have a counterpart in photography, and each technique of observation must have its equivalent in photographic recording. Hence photographs produced by modern normal and wide angle fundus cameras show the appearances of direct and indirect ophthalmoscopy in a satisfactory way.

For biomicroscopy of the fundus by means of contact lenses an adequate photographic recording is also desirable. The technique of biomicroscopy, particularly of the fundus, demands considerable skill on the part of the examiner and special expertise in the handling of the apparatus. The picture of the morphology of the fundus is built up in the examiner's mind by numerous impressions perceived one after the other by means of different adjustments of microscope and slit-lamp.

\section{Illumination}

A. Vogt' reported in 1930 on four methods of illumination for the examination of the anterior segment, which can likewise be used for the fundus as follows: focal illumination (broad beam and optical section by narrow beam); retroillumination; specular reflection; and indirect lateral illumination.

Having achieved a clear image by means of different methods of illumination and optical adjustments, the examiner has to select a few settings for the Correspondence to Dr P Kenyeres, Krankenhaus, A2130 Mistelbach, Austria. photographic recording which are as typical as possible. This is usually a task for the expert opthalmologist, because a photographer seldom has the knowledge and skill necessary for the taking of such photographs. But the most important prerequisite is to use appropriate technical equipment. This is necessary so that all methods of illumination used in the examination can also be used for the photography.

There is no problem at all in taking pictures with focal illumination of the broad beam, but photography of the narrow optical section is less satisfactory, because in this case it is impossible to relate the slit image to a definite area of the fundus. By biomicroscopy the examiner can locate the image of the optical section by changing from a broad to a narrow beam. For photographic recording it is preferable to take photographs of the thin optical section within an illuminated surrounding area. Retroillumination is especially suited for the demonstration of structures of the vitreous body or tissue parts that are detached from their plane. Curvature and regularity of shining planes are best evaluated by recording specular reflections at zones of discontinuity. Furthermore, this helps to render the narrow optical section more distinctly. Indirect lateral illumination is important for the identification of cysts and facilitates the differentiation of solid and semitransparent changes.

Slit-lamp photography of the ocular fundus is based on the same technical equipment as that used in the corresponding photorecording of the anterior segment - that is, a microscope with a beam splitter for simultaneous observation and photography on 766 


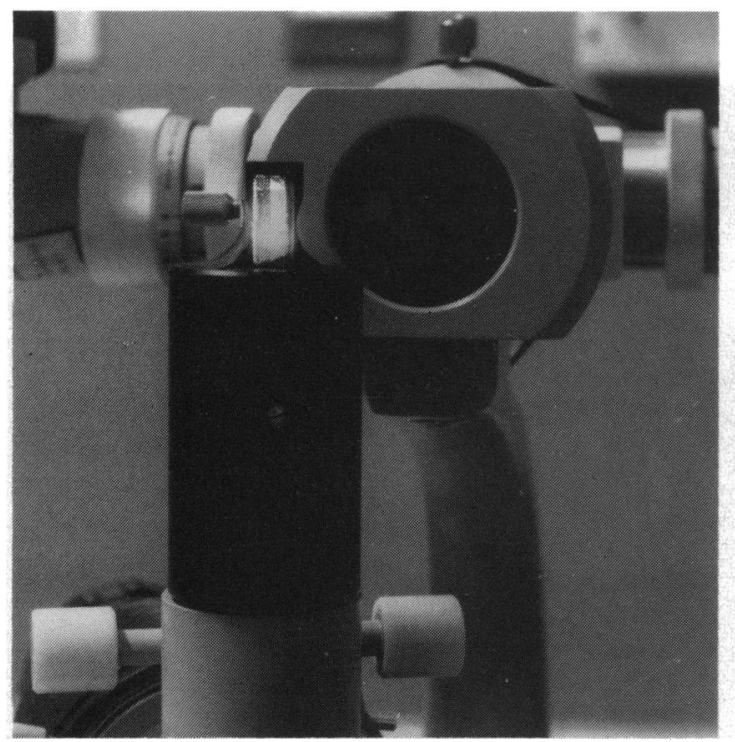

Fig. 1 Illumination splitter.

the one hand and a slit illumination of special luminar density, usually provided by an electronic flashlight, on the other.

The equipment required for additional illumination of the surrounding area, as commonly used for the photography of the anterior segment, is unsuitable for the ocular fundus, because the light cannot get there. In this case an illumination-splitter (Fig. 1), introduced by $\mathrm{us}^{2}$ and produced by Zeiss as a prototype, enables us to project both the slit light and the surrounding illumination (in the shape of two broad slit images) on to the fundus. In the photographic slit-lamp the collecting system focuses both the light of the lamp's filament and also that of the electronic flash tube into the illuminating lens. This in turn refracts the light into the eye under examination. Of the light present in the plane of the illuminating lens only a small part is used for the slit image; the greater part is held back by a slit-shaped shutter in order to render the necessary focal depth to the optical section. Part of this cut off light can be used for the surrounding illumination.

In the case of our illumination splitter the light producing the slit passes through the middle part of the illuminating lens as usual. In front of the rest of the illuminating lens the two halves of a +2.5 dioptre cylindrical lens are placed (Fig. 2). So in the case of fundus examination, two further images of the light slit are generated in front of the slit image. These overlap with the main slit image approximately in the pupillary plane. The bundle of rays then diverge and finally produce two rectangular light fields in the

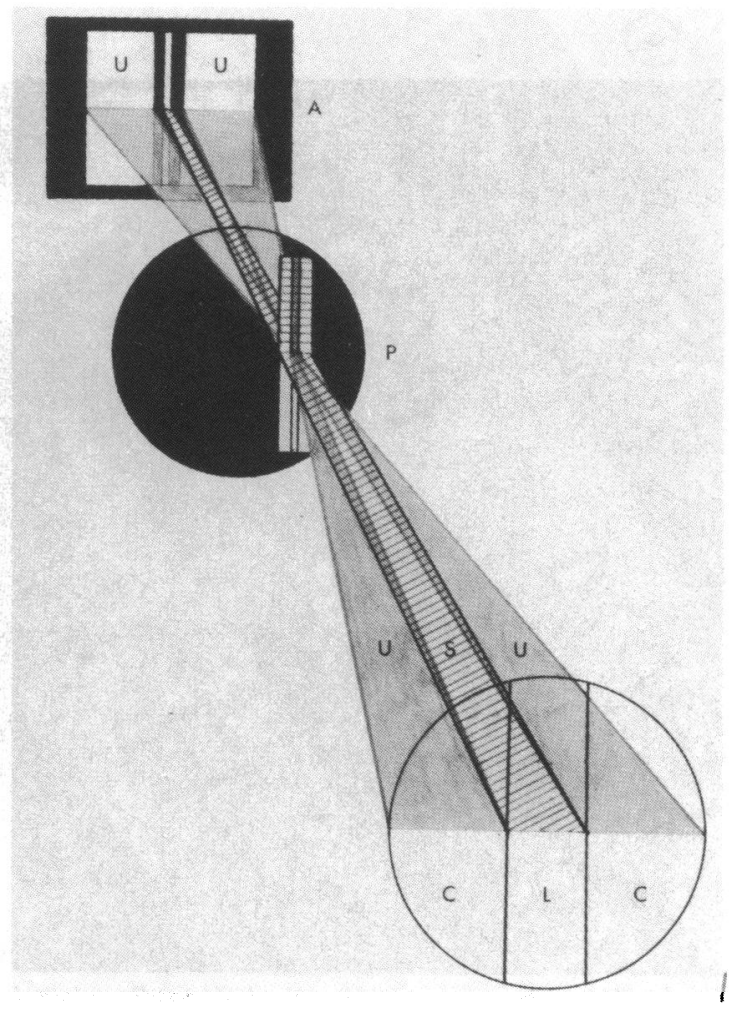

Fig. 2 Paths of rays of slit and surrounding illumination from the illuminating lens $\mathrm{L}$ to the plane of the light slit $\mathrm{A}$. If the slit light is focused on the retina by a contact lens, $\mathrm{P}$ corresponds with the point at which the rays pass through the pupil. U represents the surrounding illumination. C stands for two halves of a cylindrical lens.

plane of the main slit image - that is, the plane of the occular fundus. These two light fields light up the environment of the narrow optical section and permit its orientation. The broad slit images at both sides of the precisely focused narrow slit image are not exactly focused. A small interval between the narrow optical section and the surrounding illumination allows better recognition of delicate details in the area of the optical section (Fig. 3).

\section{Stereoscopic view}

With the sophisticated methods of ocular fundus biomicroscopy the stereoscopic view substantially improved the precise optical resolution of the observed structures. Consequently, for photographic recording, the use of stereophotography is also of crucial importance. It is done without any difficulties by insertion of light splitters connected with cameras in both barrels of the microscope. At the same time 


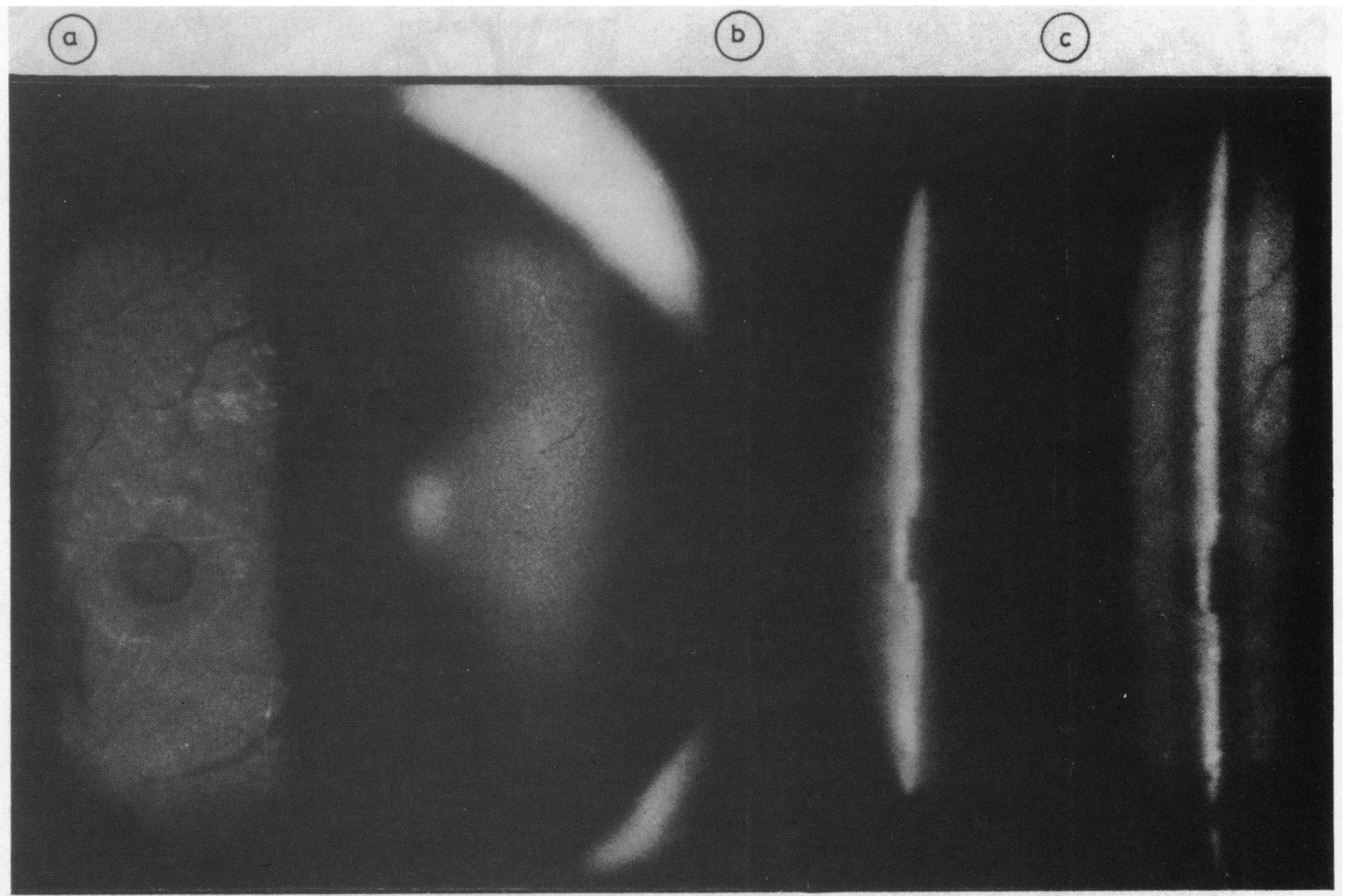

Fig. 3 Through-and-through macular hole. (a) Broad slit. (b) Narrow slit-optical section without surrounding

illumination. (c) Narrow slit-optical section, with surrounding illumination (provided by illumination splitter).

this complicates the focusing, because now the illumination path of the rays as well as two observation bundles of rays have to be located side by side within the pupil. ${ }^{3}$ In this case the ability to overlap the paths of rays of slit and surrounding illumination in the pupillary zone is an exceptional help. The biomicroscopy of the peripheral fundus is done via the Goldmann three-mirror contact lens. The examination of the extreme periphery of the fundus in addition requires the indentation of the bulbar wall. Both techniques can likewise be performed photographically. In the case of stereophotography the picturing of the temporal and nasal fundus periphery is particularly complicated, because the pupillary image is deformed to a longitudinal oval. It is often impossible therefore to position the paths of both illumination and observation rays within the short diameter of the oval pupillary image.

The use of a slit-lamp for patients examined in the supine position, ${ }^{5}$ however, enables the satisfactory binocular biomicroscopical examination of the whole lateral fundus periphery. For the photography of these cases a specially adapted operation microscope can be used.

\section{References}

1 Vogt A. Lehrbuch und Atlas der Spaltlampenmikroskopie des lebenden Auges. Erster Teil. Berlin: Springer, 1930.

2 Kenyeres P. Zur Umfeldbeleuchtung bei der Spaltampenphotographie. Graefes Arch Klin Exp Ophthalmol 1972; 184: 262-6.

3 Kenyeres P. Stereophotographic des optischen Schnittes der Spaltlampc. Graefes Arch Klin Exp Ophthalmol 1964; 167: 446-51.

4 Slezak H, Kenyeres P. Photographische Dokumentation der extremen Fundusperipherie. München: Bergmann, 1977.

5 Slezak H. Eine Spaltlampe zur Untersuchung Liegender. Graefes Arch Klin Exp Ophthalmol 1973; 186: 283-6.

Accepted for publication 16 January 1986. 